\title{
Thermodynamics of coupled identical oscillators within the path-integral formalism
}

\author{
F. Brosens and J. T. Devreese* \\ Departement Natuurkunde, Universitaire Instelling Antwerpen, Universiteit Antwerpen, Universiteitsplein 1, B-2610 Antwerpen, Belgium \\ L. F. Lemmens \\ Departement Natuurkunde, Rijksuniversitair Centrum Antwerpen, Universiteit Antwerpen, Groenenborgerlaan 171, B-2020 Antwerpen, \\ Belgium \\ (Received 26 July 1996)
}

\begin{abstract}
A generalization of symmetrized density matrices in combination with the technique of generating functions allows one to calculate the partition function of identical particles in a parabolic confining well. Harmonic two-body interactions (repulsive or attractive) are taken into account. Also the influence of a homogeneous magnetic field, introducing anisotropy in the model, is examined. Although the theory is developed for fermions and bosons, special attention is paid to the thermodynamic properties of bosons and their condensation. [S1063-651X(97)07801-X]
\end{abstract}

PACS number(s): 05.30.-d, 03.75.Fi, 32.80.Pj

\section{INTRODUCTION}

The study of the density matrices of identical particles (bosons or fermions) moving freely in a box [1] is generalized in this paper to the case of identical particles in a parabolic confinement potential with either harmonic interactions between the particles or an anisotropy induced by a homogeneous magnetic field on top of the parabolic confinement. This model, giving rise to repetitive Gaussian integrals, allows one to derive an analytical expression for the generating function of the partition function. For an ideal gas of noninteracting particles in a parabolic well, this generating function coincides with the grand-canonical partition function. With interactions, the calculation of this generating function circumvents the constraints on the summation over the cycles of the permutation group. Moreover, it allows one to calculate the canonical partition function recursively for the system with harmonic two-body interactions. The theory is developed both for fermions and for bosons. In view of the recent interest in Bose-Einstein condensation in a trap [2-4], more attention has been paid to the boson case in the discussion of the results. The model system discussed here has already been studied in the context of quantum dots with operator techniques, and the eigenvalues and eigenstates were calculated including the effect of harmonic two-body interactions and in the presence of a magnetic field [5]. However, to the best of our knowledge neither the boson case nor the thermodynamics seems to have been analyzed previously. It should also be mentioned that the idea of first expanding the Hilbert space to the configuration space and then projecting onto the appropriate subspace by grouptheoretical means has been used recently $[6,7]$ in the context of quantum dots to study the ground-state correlations for fermions and bosons.

Another motivation to perform the present analytical cal-

\footnotetext{
*Also at Rijksuniversitair Centrum Antwerpen, Universiteit Antwerpen, Groenenborgerlaan 171, B-2020 Antwerpen, Belgium and Technische Universiteit Eindhoven, The Netherlands.
}

culations lies in our path-integral formulation of the density matrices for $N$ particles [8-10]. Indeed, particles in a parabolic potential are a favorite testing ground for the pathintegral method [11-13]. It should be noted that for our formulation the existence of a positive measure over a welldefined domain in the $\mathrm{R}^{3 N}$ configuration space is essential in view of any algorithmic approach to the problem. In the present paper, which is in essence analytical, integrations over the configuration space are performed. The reason is that the extension of the state space to the configuration space makes the Gaussian integrals tractable. The permutation symmetry leads to summations over the cycles that are performed using the generating function technique, which is one of the main results of the present paper.

The model of $N$ identical particles in a parabolic well, in the presence of a magnetic field and with harmonic repulsive or attractive two-body interactions, has its intrinsic value since it constitutes an exactly soluble idealization of atoms in a magnetic trap. It should be stressed that the association of identical particles with each three oscillator degrees of freedom makes the model three dimensional. Without BoseEinstein or Fermi-Dirac statistics, i.e., for "distinguishable" particles, the model is equivalent with $3 N$ one-dimensional oscillators because each degree of freedom decouples in such a way that there is no difference in statistical behavior between $3 N$ one-dimensional (1D) oscillators and $N$ 3D oscillators [14].

This paper is organized as follows. The calculation technique is explained in the next section. In Sec. III we repeat the same calculation for the model with a homogeneous magnetic field. In Sec. IV bosons in three dimensions are analyzed in some detail. In Sec. V the conclusions are given.

\section{HARMONICALLY INTERACTING IDENTICAL PARTICLES IN A PARABOLIC WELL}

In this section we calculate the partition function of $N$ identical particles with the following Lagrangian including one-body and two-body potentials: 


$$
\begin{gathered}
L=\frac{1}{2} \sum_{j=1}^{N} \dot{\mathbf{r}}_{j}^{2}-V_{1}-V_{2}, \quad V_{1}=\frac{\Omega^{2}}{2} \sum_{j=1}^{N} \mathbf{r}_{j}^{2}, \\
V_{2}=-\frac{\omega^{2}}{4} \sum_{j, l=1}^{N}\left(\mathbf{r}_{j}-\mathbf{r}_{l}\right)^{2} .
\end{gathered}
$$

(Atomic units are used.) The potentials can be rewritten in terms of the center-of-mass coordinate $\mathbf{R}$ and coordinates $\mathbf{u}_{j}$ describing the coordinates of the particles measured from the center of mass

$$
\mathbf{R}=\frac{1}{N} \sum_{j=1}^{N} \mathbf{r}_{j}, \quad \mathbf{u}_{j}=\mathbf{r}_{j}-\mathbf{R}
$$

from which

$$
V_{1}+V_{2}=V_{\text {c.m. }}+V, \quad V_{\text {c.m. }}=\frac{1}{2} N \Omega^{2} \mathbf{R}^{2}, \quad V=w^{2} \sum_{j=1}^{N} \mathbf{u}_{j}^{2},
$$

with

$$
w=\sqrt{\Omega^{2}-N \omega^{2}} .
$$

The requirement that $w$ has to be positive expresses the stability condition that the confining potential has to be strong enough to overcome the repulsion between the particles. If a harmonic interparticle attraction is considered, the eigenfrequency $w$ would become $w=\sqrt{\Omega^{2}+N \omega^{2}}$ and no stability condition has to be imposed on the confining potential. Notice that these transformations diagonalize neither the Lagrangian nor the Hamiltonian because the coordinates $\mathbf{u}_{j}$ are not independent of the center-of-mass coordinate.

Since the system consists, in each direction, of one degree of freedom with frequency $\Omega$ and $N-1$ degrees of freedom with frequency $w$, the propagator

$$
\begin{aligned}
& K_{D}\left(\mathbf{r}_{1}^{\prime \prime}, \ldots, \mathbf{r}_{N}^{\prime \prime}, \beta \mid \mathbf{r}_{1}^{\prime}, \ldots, \mathbf{r}_{N}^{\prime}, 0\right) \\
& \quad \equiv\left\langle\mathbf{r}_{1}^{\prime \prime}, \ldots, \mathbf{r}_{N}^{\prime \prime}\left|e^{-\beta H}\right| \mathbf{r}_{1}^{\prime}, \ldots, \mathbf{r}_{N}^{\prime}\right\rangle_{D}
\end{aligned}
$$

for distinguishable particles (indicated by the subscript $D$ for 3 dimensions and $d$ in 1 dimension) can be calculated from the action expressed in the imaginary time variable and it is of course a product of the propagators $K_{d}$ per component:

$$
\begin{aligned}
& K_{D}\left(\mathbf{r}_{1}^{\prime \prime}, \ldots, \mathbf{r}_{N}^{\prime \prime}, \beta \mid \mathbf{r}_{1}^{\prime}, \ldots, \mathbf{r}_{N}^{\prime}, 0\right) \\
& \quad=K_{d}\left(\overrightarrow{x^{\prime \prime}}, \beta \mid \vec{x}, 0\right) K_{d}\left(\vec{y}^{\prime \prime}, \beta \mid \vec{y}, 0\right) K_{d}\left(\overrightarrow{z^{\prime \prime}}, \beta \mid \vec{z}, 0\right),
\end{aligned}
$$

where the column vector $\bar{x}$ contains the $x$ components of the particles, i.e., $\bar{x}^{T}=\left(x_{1}, \ldots, x_{N}\right)$ and similarly for $\bar{y}$ and $\bar{z}$. Knowing the propagator $K\left(x^{\prime \prime}, \beta \mid x^{\prime}, 0\right)_{\varpi}$ of a single harmonic oscillator with frequency $\varpi$

$$
\begin{aligned}
K\left(x_{\beta}, \beta \mid x_{0}, 0\right)_{\varpi}= & \sqrt{\frac{\varpi}{2 \pi \sinh \varpi \beta}} \\
& \times \exp \left\{-\frac{\varpi}{2} \frac{\left(x_{\beta}^{2}+x_{0}^{2}\right) \cosh \varpi \beta-2 x_{\beta} x_{0}}{\sinh \sigma \beta}\right\},
\end{aligned}
$$

one finds, for the one-dimensional propagator $K_{d}$ of the $N$ distinguishable oscillators in the interacting system,

$$
\begin{aligned}
K_{d}\left(\overrightarrow{x^{\prime \prime}}, \beta \mid \vec{x}, 0\right)= & \frac{K\left(\sqrt{N} X^{\prime \prime}, \beta \mid \sqrt{N} X^{\prime}, 0\right)_{\Omega}}{K\left(\sqrt{N} X^{\prime \prime}, \beta \mid \sqrt{N} X^{\prime}, 0\right)_{w}} \\
& \times \prod_{j=1}^{N} K\left(x_{j}^{\prime \prime}, \beta \mid x_{j}^{\prime}, 0\right)_{w},
\end{aligned}
$$

where the factor $\sqrt{N}$ in $\sqrt{N} X^{\prime \prime}$ accounts for the mass $N$ (in atomic units) of the center. The denominator in Eq. (2.8) compensates for the fact that $N-1$ instead of $N$ degrees of freedom of frequency $w$ are available. The three-dimensional propagator $K_{D}$ [Eq. (2.5)] for $N$ distinguishable oscillators of the interacting system is, according to Eqs. (2.6) and (2.8), given by

$$
\begin{aligned}
K_{D}\left(\overrightarrow{\mathbf{r}}^{\prime \prime}, \beta \mid \overline{\mathbf{r}}^{\prime}, 0\right)= & \frac{K\left(\sqrt{N} \mathbf{R}^{\prime \prime}, \beta \mid \sqrt{N} \mathbf{R}^{\prime}, 0\right)_{\Omega}}{K\left(\sqrt{N} \mathbf{R}^{\prime \prime}, \beta \mid \sqrt{N} \mathbf{R}^{\prime}, 0\right)_{w}} \\
& \times \prod_{j=1}^{N} K\left(\mathbf{r}_{j}^{\prime \prime}, \beta \mid \mathbf{r}_{j}^{\prime}, 0\right)_{w},
\end{aligned}
$$

$K\left(\mathbf{r}_{j}^{\prime \prime}, \beta \mid \mathbf{r}_{j}^{\prime}, 0\right)_{w}$

$$
=K\left(x_{j}^{\prime \prime}, \beta \mid x_{j}^{\prime}, 0\right)_{w} K\left(y_{j}^{\prime \prime}, \beta \mid y_{j}^{\prime}, 0\right)_{w} K\left(z_{j}^{\prime \prime}, \beta \mid z_{j}^{\prime}, 0\right)_{w},
$$

where $\overline{\mathbf{r}}$ denotes a point in the configuration space $\mathbb{R}^{3 N}$, i.e., $\overline{\mathbf{r}}^{T} \equiv\left(\left(x_{1}, y_{1}, z_{1}\right), \ldots,\left(x_{N}, y_{N}, z_{N}\right)\right)$. The symmetrized density matrix $K_{I}$ for $3 \mathrm{D}$ identical particles (indicated by the subscript $I$ ) can be obtained by using the following projection, with $P$ denoting the permutation matrix:

$$
K_{I}\left(\overline{\mathbf{r}}^{\prime \prime}, \beta \mid \overline{\mathbf{r}}^{\prime}, 0\right)=\frac{1}{N !} \sum_{p} \xi^{p} K_{D}\left(P \overline{\mathbf{r}}^{\prime \prime}, \beta \mid \overline{\mathbf{r}}^{\prime}, 0\right),
$$

where $\xi=+1$ for bosons and $\xi=-1$ for fermions. It should be emphasized that $P$ acts on the particle indices, not on the components of $\mathbf{r}$ separately. The partition function is then readily obtained by integrating over the configuration space

$$
Z_{I}=\int d \overline{\mathbf{r}} K_{I}(\overline{\mathbf{r}}, \beta \mid \overline{\mathbf{r}}, 0)=\int d \overline{\mathbf{r}}_{N !}^{1} \sum_{p} \xi^{p} K_{D}(P \overline{\mathbf{r}}, \beta \mid \overline{\mathbf{r}}, 0) .
$$

The remaining part of this section will be devoted to the explicit evaluation of this integral for the partition function. The integration proceeds in three stages: the first stage deals with the center-of-mass treatment, the second one concerns the cyclic decomposition, and in the third step the summation over the cycles will be performed.

\section{A. Center of mass}

The center-of-mass coordinate $\mathbf{R}$ not only depends on the coordinates of all the particles, but it also has its own propagator. Therefore, substituting $\mathbf{R}$ by its expression in terms of the particle positions and then performing the integration seems not to be the most adequate way to deal with the integration over the configuration space. Instead, the follow- 
ing identity is used for the formal treatment of $\mathbf{R}$ as an independent coordinate, at the expense of additional integrations:

$$
\int d \overline{\mathbf{r}} f\left(\overline{\mathbf{r}}, \frac{1}{N} \sum_{j=1}^{N} \mathbf{r}_{j}\right)=\int d \mathbf{R} \int d \overline{\mathbf{r}} f(\overline{\mathbf{r}}, \mathbf{R}) \delta\left(\mathbf{R}-\frac{1}{N} \sum_{j=1}^{N} \mathbf{r}_{j}\right) .
$$

Fourier transformation of the $\delta$ function then leads to

$$
\begin{aligned}
& \int d \overline{\mathbf{r}} f\left(\overline{\mathbf{r}}, \frac{1}{N} \sum_{j=1}^{N} \mathbf{r}_{j}\right) \\
& =\int d \mathbf{R} \int \frac{d \mathbf{k}}{(2 \pi)^{3}} e^{i \mathbf{k} \cdot \mathbf{R}} \int d \overline{\mathbf{r}} f(\overline{\mathbf{r}}, \mathbf{R}) e^{-i \overline{\mathbf{k}} \cdot \overline{\mathbf{r}}},
\end{aligned}
$$

where $\overline{\mathbf{k}}^{T}=(k / N)((1,1,1), \ldots,(1,1,1))$ is a $3 N$-dimensional row vector. Applying this transformation to the partition function $Z_{I}$ and rearranging the factors one obtains

$$
\begin{aligned}
Z_{I}= & \int d \mathbf{R} \int \frac{d \mathbf{k}}{(2 \pi)^{3}} e^{i \mathbf{k} \cdot \mathbf{R}} \frac{K(\sqrt{N} \mathbf{R}, \beta \mid \sqrt{N} \mathbf{R}, 0)_{\Omega}}{K(\sqrt{N} \mathbf{R}, \beta \mid \sqrt{N} \mathbf{R}, 0)_{w}} \\
& \times \int d \overline{\mathbf{r}} \frac{1}{N !} \sum_{p} \xi^{p} \prod_{j=1}^{N} K\left((P \mathbf{r})_{j}, \beta \mid \mathbf{r}_{j}, 0\right)_{w} e^{-i \mathbf{k} \cdot \mathbf{r}_{j} / N} .
\end{aligned}
$$

This transformation makes $\mathbf{R}$ independent of the particle positions relative to the center of mass. The real dependence on the relative positions is reintroduced by the Fourier transform. It should be noted that the explicit dependence of the propagator (2.9) on $\mathbf{R}$, and the presence of the factor $e^{-i \mathbf{k} \cdot \mathbf{r}_{j} / N}$ are consequences of the two-body interactions.

The next step is to rewrite the sum over the permutations as a sum over all possible cycles. This will be done in the next subsection. An excellent example of such a decomposition into cycles has been given by Feynman [1] for a system of noninteracting particles in a box.

\section{B. Cyclic decomposition}

A permutation can be broken up into cycles. Suppose that a particular permutation contains $M_{l}$ cycles of length $l$. The positive integers $M_{l}$ and $l$ then have to satisfy the constraint

$$
\sum_{l} l M_{l}=N
$$

Furthermore, the number $M\left(M_{1}, \ldots, M_{N}\right)$ of cyclic decompositions with $M_{1}$ cycles of length $1, \ldots, M_{l}$ cycles of length $l, \ldots$ is known to be

$$
M\left(M_{1}, \ldots, M_{N}\right)=\frac{N !}{\Pi_{l} M_{l} ! l^{M_{l}}} .
$$

A cycle of length $l$ will be obtained from $l-1$ permutations. Therefore, the sign factor $\xi^{p}$ can be decomposed as

$$
\xi^{p}=\prod_{l} \xi^{(l-1) M_{l}}
$$

Combining these results originating from the permutation symmetry, one obtains

$$
\begin{gathered}
Z_{I}=\int d \mathbf{R} \int \frac{d \mathbf{k}}{(2 \pi)^{3}} e^{i \mathbf{k} \cdot \mathbf{R}} \frac{K(\sqrt{N} \mathbf{R}, \beta \mid \sqrt{N} \mathbf{R}, 0)_{\Omega}}{K(\sqrt{N} \mathbf{R}, \beta \mid \sqrt{N} \mathbf{R}, 0)_{w}} \\
\times \sum_{M_{1}, \ldots, M_{N}} \prod_{l} \frac{\xi^{(l-1) M_{l}}}{M_{l} ! l^{M_{l}}}\left[\mathcal{K}_{l}(\mathbf{k})\right]^{M_{l}}, \\
\mathcal{K}_{l}(\mathbf{k})=\int d \mathbf{r}_{l+1} \int d \mathbf{r}_{l} \cdots \int d \mathbf{r}_{1} \delta\left(\mathbf{r}_{l+1}-\mathbf{r}_{1}\right) \\
\quad \times \prod_{j=1}^{N} K\left(\mathbf{r}_{j+1}, \beta \mid \mathbf{r}_{j}, 0\right)_{w} e^{-i \mathbf{k} \cdot \mathbf{r}_{j} / N} .
\end{gathered}
$$

The $\delta$ function expresses that the decomposition is cyclic. It is obvious that

$$
\mathcal{K}_{l}(\mathbf{k})=\mathcal{K}_{l}^{(1 \mathrm{D})}\left(k_{x}\right) \mathcal{K}_{l}^{(1 \mathrm{D})}\left(k_{y}\right) \mathcal{K}_{l}^{(1 D)}\left(k_{z}\right)
$$

which allows one to analyze $\mathcal{K}_{l}(\mathbf{k})$ from its one-dimensional constituents

$$
\begin{aligned}
\mathcal{K}_{l}^{(1 \mathrm{D})}\left(k_{x}\right)= & \int d x_{l+1} \int d x_{l} \cdots \int d x_{1} \delta\left(x_{l+1}-x_{1}\right) \\
& \times \prod_{j=1}^{N} K\left(x_{j+1}, \beta \mid x_{j}, 0\right)_{w} e^{-i k_{x} x_{j} / N} .
\end{aligned}
$$

Using the semigroup property [15] of the harmonicoscillator propagator $K\left(x_{j+1}, \beta \mid x_{j}, 0\right)_{w}$, all integrations but one can be performed

$\mathcal{K}_{l}^{(1 \mathrm{D})}\left(k_{x}\right)=\int d x K(x, l \beta \mid x, 0)_{w} \exp \left(-\int_{0}^{l \beta} d \tau f_{x}(\tau) x(\tau)\right)$,

where

$$
f_{x}(\tau)=i \frac{k_{x}}{N} \sum_{j=0}^{l-1} \delta(\tau-j \beta)
$$

The integral (2.23) is the propagator $K_{w, f}$ of a driven harmonic oscillator with the Lagrangian

$$
L_{w, f_{x}}=\frac{1}{2} \dot{x}^{2}-\frac{1}{2} w^{2} x^{2}+f_{x}(\tau) x
$$

studied in $[11,16]$. It should be noted again that without twobody interactions the driving force (2.24) is lacking. Taking over the result from [16] and integrating over the configuration space, one obtains 


$$
\begin{aligned}
Z_{w, f_{x}}(\beta)= & d x K_{w, f_{x}}(x, \beta \mid x, 0) \\
= & \frac{1}{2 \sinh \frac{1}{2} \beta w} \exp \left(\frac{1}{2} \int_{0}^{\beta} d \tau \int_{0}^{\beta} d \sigma \frac{f_{x}(\tau) f_{x}(\sigma)}{2 w}\right. \\
& \left.\times \frac{\cosh \left[\left(\frac{\beta}{2}-|\tau-\sigma|\right) w\right]}{\sinh \frac{1}{2} \beta w}\right)
\end{aligned}
$$

After straightforward algebra one obtains, for the 1D function $\mathcal{K}_{l}^{(1 \mathrm{D})}\left(k_{x}\right)$,

$$
\mathcal{K}_{l}^{(1 \mathrm{D})}\left(k_{x}\right)=\frac{1}{2 \sinh \frac{1}{2} l \beta w} \exp \left(-\frac{l}{4 N^{2}} \frac{k_{x}^{2}}{w} \frac{1+e^{-\beta w}}{1-e^{-\beta w}}\right)
$$

and for its 3D extension

$$
\mathcal{K}_{l}(\mathbf{k})=\left(\frac{1}{2 \sinh \frac{1}{2} l \beta w}\right)^{3} \exp \left(-\frac{l}{4 N^{2}} \frac{k^{2}}{w} \frac{1+e^{-\beta w}}{1-e^{-\beta w}}\right) .
$$

Using Eq. (2.16) one then is left with a sixfold integral for the partition function

$$
\begin{aligned}
Z_{I}= & \int d \mathbf{R} \int \frac{d \mathbf{k}}{(2 \pi)^{3}} e^{i \mathbf{k} \cdot \mathbf{R}} \frac{K(\sqrt{N} \mathbf{R}, \beta \mid \sqrt{N} \mathbf{R}, 0)_{\Omega}}{K(\sqrt{N} \mathbf{R}, \beta \mid \sqrt{N} \mathbf{R}, 0)_{w}} \\
& \times \exp \left(-\frac{1}{4 N} \frac{k^{2}}{w} \frac{1+e^{-\beta w}}{1-e^{-\beta w}}\right) \\
& \times \sum_{M_{1}, \ldots, M_{N}} \prod_{l} \frac{\xi^{(l-1) M_{l}}}{M_{l} ! l^{M_{l}}}\left(\frac{1}{2 \sinh _{\frac{1}{2}} l \beta w}\right)^{3 M_{l}} .
\end{aligned}
$$

Both the integrations over $\mathbf{k}$ and $\mathbf{R}$ are Gaussian, leading to the following series for $Z_{I}$ :

$$
\begin{gathered}
Z_{I}=\left(\frac{\sinh \frac{1}{2} \beta w}{\sinh \frac{1}{2} \beta \Omega}\right)^{3} Z_{I}(N), \\
Z_{I}(N) \equiv \sum_{M_{1}, \ldots, M_{N}} \prod_{l} \frac{\xi^{(l-1) M_{l}}}{M_{l} ! l^{M_{l}}}\left(\frac{e^{-(1 / 2) l \beta w}}{1-e^{-l \beta w}}\right)^{3 M_{l}} .
\end{gathered}
$$

Without two-body interactions $(w=\Omega), \mathbb{Z}_{I}(N)$ is the partition function of a set of identical oscillators. The partition function $Z_{I}$ differs from it only by a center-of-mass correction and the actual values of $w$. The remaining summation over the cycles involves the constraint (2.16), which, however, can be removed by the use of the generating function technique, which will be considered in the next subsection.

\section{Generating function}

Concentrating on the explicit dependence of $\mathbb{Z}_{I}(N)$ on $N$ (with $w$ considered as a parameter), one can construct the generating function

$$
\Xi(u)=\sum_{N=0}^{\infty} \mathbb{Z}_{I}(N) u^{N},
$$

with $\mathbb{Z}_{I}(0)=1$ by definition. The partition function $\mathbb{Z}_{I}(N)$ can then be obtained by taking the appropriate derivatives of $\Xi(u)$ with respect to $u$, assuming that the series for $\Xi(u)$ is convergent near $u=0$ :

$$
\mathbb{Z}_{I}(N)=\left.\frac{1}{N !} \frac{d^{N}}{d u^{N}} \Xi(u)\right|_{u=0}
$$

The summation over the number of cycles with length $l$ is now unrestricted and can easily be performed:

$$
\Xi_{I}(u)=\exp \left(\sum_{l=1}^{\infty} \xi^{l-1} \frac{e^{-(3 / 2) l \beta w} u^{l}}{l\left(1-e^{-l \beta w}\right)^{3}}\right) \text {. }
$$

This series can be rewritten into the more familiar form

$$
\begin{aligned}
\Xi_{I}(u)= & \exp \left(-\xi \sum_{\nu=0}^{\infty} \frac{1}{2}(\nu+1)(\nu+2)\right. \\
& \left.\times \ln \left(1-\xi u e^{-\beta w(3 / 2+\nu)}\right)\right) .
\end{aligned}
$$

Before we study this equation in detail two remarks are in order: First, $\Xi_{I}(u)$ is the generating function of a model without two-body interactions. In that case $w=\Omega$ and $\Xi_{I}(u)$ coincides with the well-known [17-19] grandcanonical partition function of a set of identical particles in a parabolic well. Second, for the noninteracting case in one dimension [i.e., with the multiplicity factor $\frac{1}{2}(\nu+1)(\nu+2)$ replaced by 1$]$, we recover the results of Ref. [20]. To the best of our knowledge, the interacting case has not been analyzed up to now for identical particles. For distinguishable particles it has been studied before [21].

\section{Recurrence relations for the partition function}

Starting from the expression for $\Xi_{I}(u)$ derived in the preceding subsection for the interacting model, a recursion relation can be obtained for $\mathbb{Z}_{I}(N)$. Introducing

$$
b=e^{-\beta w}
$$

for brevity in the notations, we observe that

$$
\frac{d}{d u} \Xi_{I}(u)=\Xi_{I}(u) \sum_{\nu=0}^{\infty} \frac{1}{2}(\nu+1)(\nu+2) \frac{b^{3 / 2+\nu}}{1-\xi u b^{3 / 2+\nu}} .
$$

Considering next 


$$
\mathbb{Z}_{I}(N)=\left.\frac{1}{N !} \frac{d^{N-1}}{d u^{N-1}} \frac{d}{d u} \Xi(u)\right|_{u=0},
$$

the product rule and an elementary binomial expansion can be used to find

$$
\mathbb{Z}_{I}(N)=\frac{1}{N} \sum_{m=0}^{N-1} \xi^{N-m-1}\left(\frac{b^{(1 / 2)(N-m)}}{1-b^{N-m}}\right)^{3} Z_{I}(m)
$$

The corresponding one-dimensional version of this recurrence relation (indicated with the subscript $i$ to distinguish it from the $3 \mathrm{D}$ case with capital subscript) becomes

$$
Z_{i}(N)=\frac{1}{N} \sum_{m=0}^{N-1} \xi^{N-m-1} \frac{b^{(1 / 2)(N-m)}}{1-b^{N-m}} Z_{i}(m),
$$

leading to the following partition functions in closed form for one-dimensional bosons and one-dimensional fermions:

$$
\mathbb{Z}_{b}=\frac{b^{(1 / 2) N}}{\prod_{j=1}^{N}\left(1-b^{j}\right)}, \mathbb{Z}_{f}=\frac{b^{(1 / 2) N^{2}}}{\prod_{j=1}^{N}\left(1-b^{j}\right)}
$$

It is easy to check that these partition functions are the solution of the recurrence relation for $\mathbb{Z}_{i}(N)$ with $\xi=1$ for bosons and $\xi=-1$ for fermions. However, we did not find a systematic method to obtain analytical solutions of this type of recurrence relations. For example, for the 3D case we had to rely on numerical schemes, as will be discussed below. But at this stage, it is worthwhile first to consider the presence of an homogeneous magnetic field as the origin of anisotropy in our model of $N$ identical oscillators.

\section{III. $N$ IDENTICAL OSCILLATORS IN A MAGNETIC FIELD}

The Lagrangian of $N$ particles in a confining parabolic potential in the presence of a magnetic field is

$$
L_{\omega_{c}}=\frac{1}{2} \sum_{j=1}^{N}\left(\dot{\mathbf{r}}_{j}-2 \omega_{c} x_{j} \dot{y}_{j}\right)^{2}-\frac{1}{2} \Omega^{2} \sum_{j=1}^{N} \mathbf{r}_{j}^{2},
$$

where $\omega_{c}$ is the cyclotron frequency. For this model, the calculations of the preceding section can in essence be re-

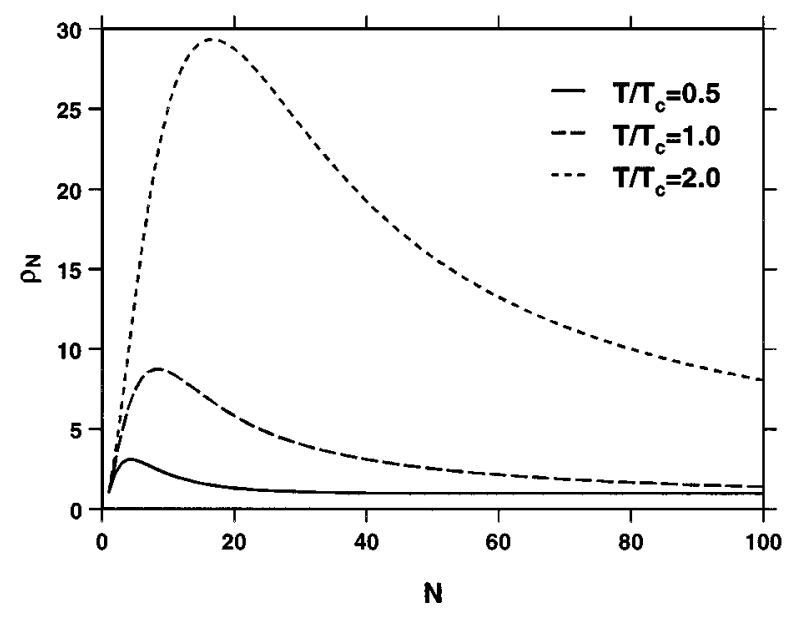

FIG. 1. Values of $\rho_{1}, \ldots, \rho_{N}$ for $N=100$ from the recurrence relation (4.3) for the three temperatures $T / T_{c}=0.5,1$, and 2 .

peated. First the propagator for distinguishable particles is calculated. The next step will be the projection on the irreducible representation of the permutation group and performing the cyclic decomposition. Then the generating function is introduced to circumvent the constraints on the partition in cycles and finally the summation over the cycles is performed.

The fact that the energy spectrum and the wave function can be calculated when harmonic interparticle interactions are included [5] indicates that the propagator and the partition function for the model in a magnetic field with two-body interaction can be obtained using our methods. The calculation technique is very demanding; therefore it seemed appropriate to illustrate the method on the basis of the simple model (3.1).

\section{A. The propagator for distinguishable particles}

For distinguishable particles, the many-particle propagator is a product of one-particle propagators. The one-particle propagator $K_{\omega_{c}}^{(1)}\left(\mathbf{r}, \beta \mid \mathbf{r}^{\prime}\right)$ of this model can be calculated by stochastic techniques [22] or by path-integral techniques [11]. The evaluation is somewhat lengthy but straightforward, resulting eventually in

$$
\begin{aligned}
K_{\omega_{c}}^{(1)}\left(\mathbf{r}, \beta \mid \mathbf{r}^{\prime}\right)= & \sqrt{\frac{\Omega}{2 \pi \sinh \beta \Omega}} \frac{s}{2 \pi \sinh \beta s} \exp \left\{-\frac{\Omega\left\{\left[z^{2}+\left(z^{\prime}\right)^{2}\right] \cosh \beta \Omega-2 z z^{\prime}\right\}}{2 \sinh \beta \Omega}\right\} \\
& \times \exp \left\{-\frac{s}{2}\left(x^{2}+y^{2}+\left(x^{\prime}\right)^{2}+\left(y^{\prime}\right)^{2}\right) \cosh \beta s-2\left(x x^{\prime}+y y^{\prime}\right) \cosh \frac{1}{2} \beta \omega \sinh \beta s\right\} \\
& \times \exp \left\{-i\left(\frac{1}{2} \omega_{c}\left(x y-x^{\prime} y^{\prime}\right)-s \frac{\sinh \frac{1}{2} \beta \omega}{\sinh \beta s}\left(y^{\prime} x-y x^{\prime}\right)\right)\right\},
\end{aligned}
$$


with the eigenfrequency $s$ given by

$$
s=\sqrt{\Omega^{2}+\frac{1}{4} \omega_{c}^{2}} .
$$

The partition function corresponding to this single-particle propagator is obtained by integrating over the configuration space

$$
\begin{aligned}
\mathbb{Z}_{\omega_{c}}^{(1)}(\beta) & =\int d \mathbf{r} K_{\omega_{c}}^{(1)}(\mathbf{r}, \beta \mid \mathbf{r}) \\
& =\left[8 \sinh \beta \frac{\Omega}{2} \sinh \beta\left(\frac{s}{2}+\frac{\omega_{c}}{4}\right) \sinh \beta\left(\frac{s}{2}-\frac{\omega_{c}}{4}\right)\right]^{-1},
\end{aligned}
$$

which coincides with the partition function of a 3D harmonic oscillator if $\omega_{c}=0$.

\section{B. The partition function and generating function for identical particles}

For $N$ identical particles, the propagator becomes

$$
K_{I, \omega_{c}}\left(\overline{\mathbf{r}}, \beta \mid \overline{\mathbf{r}}^{\prime}\right)=\frac{1}{N !} \sum_{p} \xi^{p} \prod_{j=1}^{N} K_{\omega_{c}}^{(1)}\left((P \mathbf{r})_{j}, \beta \mid \mathbf{r}_{j}^{\prime}\right),
$$

with the corresponding partition function given by

$$
\mathbb{Z}_{I, \omega_{c}}(N)=\int d \overline{\mathbf{r}} K_{I, \omega_{c}}(\overline{\mathbf{r}}, \beta \mid \overline{\mathbf{r}}) .
$$

Using the cyclic decomposition and the semigroup property as before, followed by the relaxation of the constraint (2.16) on the number of cycles, one finds for the generating function

$$
\Xi_{\omega_{c}}(u)=\exp \left(\sum_{l=1}^{\infty} \frac{\xi^{l-1}}{l} \mathbb{Z}_{\omega_{c}}^{(1)}(l \beta) u^{l}\right)
$$

Because, in this model, with a magnetic field no two-body interactions were taken into account, this expression equals the grand-canonical partition function of the model if $u=e^{\beta \mu}$ is interpreted as the fugacity, with $\mu$ the chemical potential.

\section{BOSONS IN THREE DIMENSIONS}

In the previous sections we have obtained the grandcanonical partition function of identical harmonic oscillators in a confining parabolic potential well without a magnetic field [Eq. (2.34)] and in the presence of a magnetic field [Eq. (3.7)]. For the same system the canonical partition function is obtained for an attractive as well as for a repulsive harmonic two-body interaction [Eq. (2.30)]. The expressions are given for bosons and for fermions.

The actual calculation of the thermodynamic properties or the mean-square radius of the 3D cloud of identical particles is substantially complicated by the lack of an explicit expression in closed form for the partition function. Therefore numerical methods have to be used. We concentrate this analy- sis on the boson case. For fermions a different scheme will have to be developed.

\section{A. In the absence of a magnetic field}

The recurrence relation (2.37) is not directly accessible for numerical computation, as can easily be seen by evaluating the expected dominant factor $b^{3 N / 2} / \Pi_{j=1}^{N}\left(1-b^{j}\right)^{3}$ for bosons. For a relatively low temperature and a moderate number of particles, say, $b=0.75$ and $N=1000$, this factor is as small as $1.0402 \times 10^{-182}$. We therefore isolate this factor using the following scaling for the partition function:

$$
\mathbb{Z}_{B}(N)=\sigma_{N} \frac{b^{(3 / 2) N}}{\Pi_{j=1}^{N}\left(1-b^{j}\right)^{3}},
$$

and rewrite the recurrence relation (2.37) in terms of the activity $\rho_{N}$ (see, e.g., [21]) defined as

$$
\sigma_{N}=\rho_{N} \sigma_{N-1} \Rightarrow \sigma_{N}=\sigma_{0} \prod_{j=0}^{N} \rho_{j}
$$

where $\sigma_{0}=\rho_{0} \equiv 1$ have been introduced for convenience. The recurrence relation (2.37) then becomes, after some manipulations,

$$
\rho_{N}=\frac{1}{N}\left(\frac{1-b^{N}}{1-b}\right)^{3}\left[1+\sum_{m=0}^{N-2}\left(\frac{1-b}{1-b^{N-m}}\right)^{3} \prod_{j=m+1}^{N-1} \frac{\left(1-b^{j}\right)^{3}}{\rho_{j}}\right] .
$$

The corresponding recurrence relation for the internal energy of the internal degrees of freedom becomes

$$
\begin{aligned}
\frac{\mathrm{U}_{B}(N)}{\hbar w}= & \frac{1}{N} \frac{1}{\rho_{N}}\left(\frac{1-b^{N}}{1-b}\right)^{3}\left\{\frac{3}{2} \frac{1+b}{1-b}+\frac{\mathrm{U}_{B}(N-1)}{\hbar w}\right. \\
& +\sum_{m=0}^{N-2}\left[\left(\frac{3}{2}(N-m) \frac{1+b^{N-m}}{1-b^{N-m}}+\frac{\mathrm{U}_{B}(m)}{\hbar w}\right)\right. \\
& \left.\left.\times\left(\frac{1-b}{1-b^{N-m}}\right)^{3} \prod_{j=m+1}^{N-1} \frac{\left(1-b^{j}\right)^{3}}{\rho_{j}}\right]\right\} .
\end{aligned}
$$

The temperature scale used to express $b=e^{-w / k T}$ is

$$
t=\left(\frac{N}{\zeta(3)}\right)^{-1 / 3} \frac{k T}{w} \equiv \frac{T}{T_{c}}
$$

where

$$
\zeta(3)=1.2021
$$

The recurrence relations (4.3) and (4.4) for $\rho_{1}, \ldots, \rho_{N}$ and $\mathrm{U}_{B}(1), \ldots, \mathrm{U}_{B}(N)$ can be computed very efficiently. The values of $\rho_{1}, \ldots, \rho_{N}$ for $N=100$ are shown in Fig. 1 for the three temperatures $T / T_{c}=0.5,1$, and 2 . For $T=2 T_{c}$ it turns out that $\rho_{j}$ is about 8 times larger than $\rho_{j-1}$ for $j$ approach- 


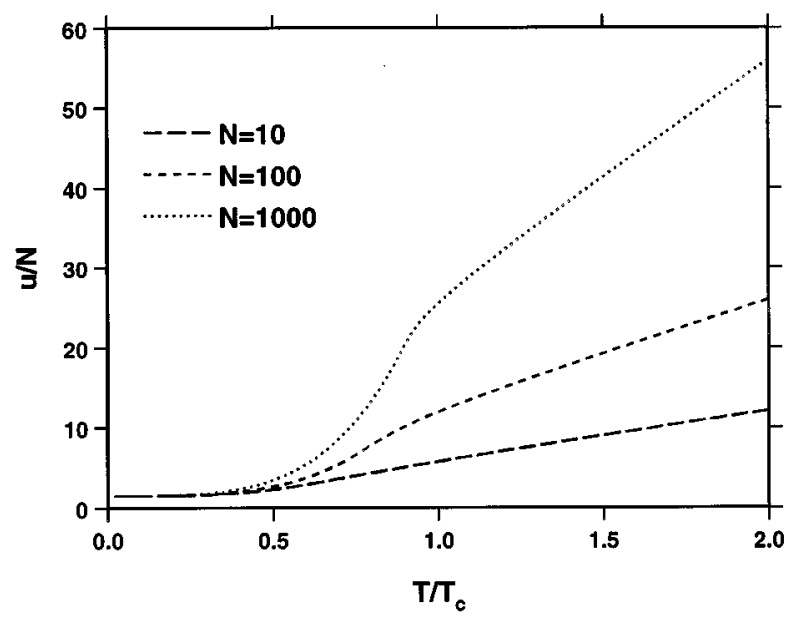

FIG. 2. Internal energy $u / N \equiv \mathrm{U}_{B}(N) / N \hbar w$ for $N=10,100$, 1000 as a function of $T / T_{c}$.

ing $N$, which makes it extremely difficult to deal numerically with the values of the partition function rather than with the proportionality factors.

Once $\rho_{N}$ is known, the internal energy $\mathrm{U}_{B}(N)$ is readily obtained from Eq. (4.4). The results for $N=10,100$, and 1000 are shown in Fig. 2. The specific heat in the canonical ensemble can also be calculated from these parameters. This is shown in Fig. 3, clearly illustrating the effect of condensation for a finite number of particles. Anticipating Sec. IV B, it should be noted that the specific heat in the canonical ensemble for $N$ particles and without repulsive two-body interactions is identical to the specific heat in the grandcanonical ensemble with the average number of particles given by the same value of $N$. The reason why we compare both ensembles without repulsive interactions is the requirement that in the grand-canonical ensemble the system should be stable for any large number of particles, which is not the case in the Gaussian model with repulsion because the confining potential can only accommodate a finite number of particles as a consequence of Eq. (2.4). Another interesting consequence of the repulsive interactions follows from the dependence of the condensation temperature $T_{c}$ on the number of particles, which obeys the following scaling law taking Eq. (2.4) into account:

$$
\begin{aligned}
T_{c}= & \frac{\sqrt{\Omega^{2}-N \omega^{2}}}{k}\left(\frac{N}{\zeta(3)}\right)^{1 / 3} \\
& \Rightarrow \frac{k T_{c}}{\Omega}\left(\frac{\omega^{2}}{\Omega^{2}} \zeta(3)\right)^{1 / 3}=\sqrt{1-\frac{N \omega^{2}}{\Omega^{2}}}\left(\frac{N \omega^{2}}{\Omega^{2}}\right)^{1 / 3} .
\end{aligned}
$$

For the case of attractive interactions and no confinement potential, $T_{c}$ is proportional to $N^{4 / 3}$. The condensation temperature for both cases is plotted in Fig. 4. For the Gaussian model, the case of harmonic attraction does not pose any problem because in this model there is no sign of an "extra" collapse due to the nature of the interaction. The only consequence seems to be that the condensation occurs at a much higher temperature than would be the case without two-body interactions or with a repulsive harmonic interaction.

\section{B. In the presence of a magnetic field}

The thermodynamic properties in the grand-canonical ensemble of an ideal Bose gas in a parabolic well and in the presence of a magnetic field can be obtained directly from Eqs. (3.4) and (3.7). Substituting $u$ by the fugacity $e^{\beta \mu}$, the Gibbs free energy $G_{\omega_{c}}$ for the boson case $(\xi=+1)$ becomes

$$
G_{\omega_{c}}=-\frac{1}{\beta} \sum_{l=1}^{\infty} \frac{1}{l} \frac{e^{l \beta[\mu-(1 / 2) \Omega-s]}}{\left(1-e^{-l \beta \Omega}\right)\left(1-e^{-l \beta\left[s+(1 / 2) \omega_{c}\right]}\right)\left(1-e^{-l \beta\left[s-(1 / 2) \omega_{c}\right]}\right)} .
$$

After a power-series expansion of the denominators, the summation over the cycle lengths $l$ can be performed:

$$
\begin{aligned}
G_{\omega_{c}}= & \frac{1}{\beta_{j, k, l=0}} \sum^{\infty} \ln \left(1-e^{\beta \mu} e^{-\beta \Omega(1 / 2+j)}\right. \\
& \left.\times e^{-\beta s(1+k+l)} e^{-(1 / 2) \beta \omega_{c}(k-l)}\right),
\end{aligned}
$$

as expected from a Bose-Einstein distribution with energy levels

$$
\epsilon_{j, k, l}=\frac{1}{2} \Omega+s+j \Omega+k\left(s+\frac{1}{2} \omega_{c}\right)+l\left(s-\frac{1}{2} \omega_{c}\right) .
$$

$$
\begin{gathered}
N=\sum_{j, k, l=0}^{\infty} n_{j, k, l}, \\
n_{j, k, l}=\frac{e^{\beta \mu} e^{-\beta \Omega(1 / 2+j)} e^{-\beta s(1+k+l)} e^{-(1 / 2) \beta \omega_{c}(k-l)}}{1-e^{\beta \mu} e^{-\beta \Omega(1 / 2+j)} e^{-\beta s(1+k+l)} e^{-(1 / 2) \beta \omega_{c}(k-l)}}
\end{gathered}
$$

and the fugacity can be eliminated in favor of the groundstate occupancy $n_{0}$,

$$
n_{0}=\frac{e^{\beta \mu} e^{-(1 / 2) \beta \Omega} e^{-\beta s}}{1-e^{\beta \mu} e^{-(1 / 2) \beta \Omega} e^{-\beta s}} \Rightarrow e^{\beta \mu}=\alpha e^{\beta[(1 / 2) \Omega+s]}
$$

with

$$
\alpha \equiv n_{0} /\left(n_{0}+1\right) .
$$




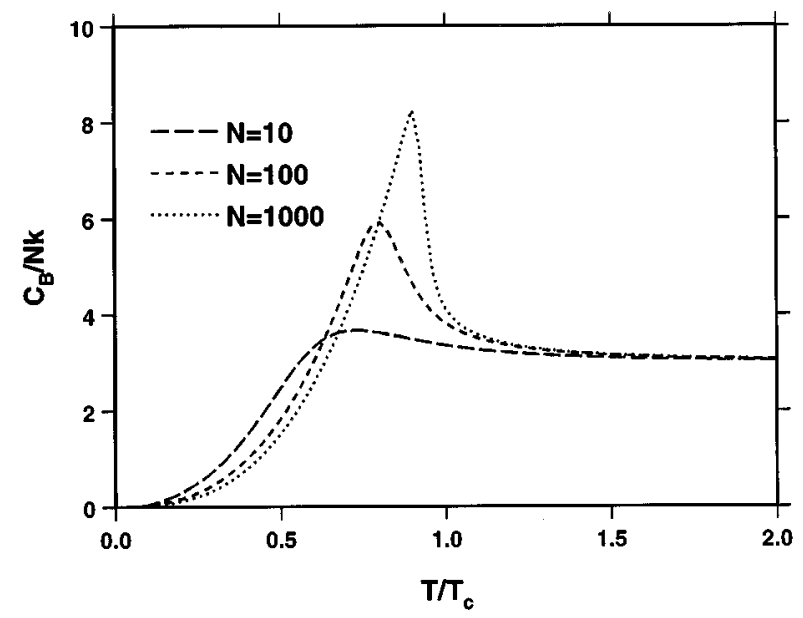

FIG. 3. Specific heat $C_{B} / N k$ per particle in units of the Boltzmann constant $k$ for $N=10,100,1000$ as a function of $T / T_{c}$.

Restoring the cyclic summation for reasons of numerical convergence, one obtains for the average number of particles

$$
\begin{aligned}
N=\sum_{l=1}^{\infty} \frac{\alpha^{l}}{D_{l}}, \quad D_{l} \equiv & \left(1-e^{-l \beta \Omega}\right)\left(1-e^{-l \beta\left[s+(1 / 2) \omega_{c}\right]}\right) \\
& \times\left(1-e^{-l \beta\left[s-(1 / 2) \omega_{c}\right]}\right)
\end{aligned}
$$

which can be cast in the form of the numerically tractable series

$$
N=\frac{\alpha}{1-\alpha}+\sum_{l=1}^{\infty} \alpha^{l}\left(\frac{1}{D_{l}}-1\right)
$$

For given $N$, standard numerical techniques can be used to determine $\alpha \in[0,1]$ and hence the ground-state occupancy $n_{0}$, which is shown in Fig. 5 for $\omega_{c}=0$ and in Fig. 6 for $\omega_{c} / \Omega=5$ as a function of $T / T_{c}$ for several values of $N$. It turns out that the parabolic well is more important than the anisotropy due to the presence of the magnetic field. The

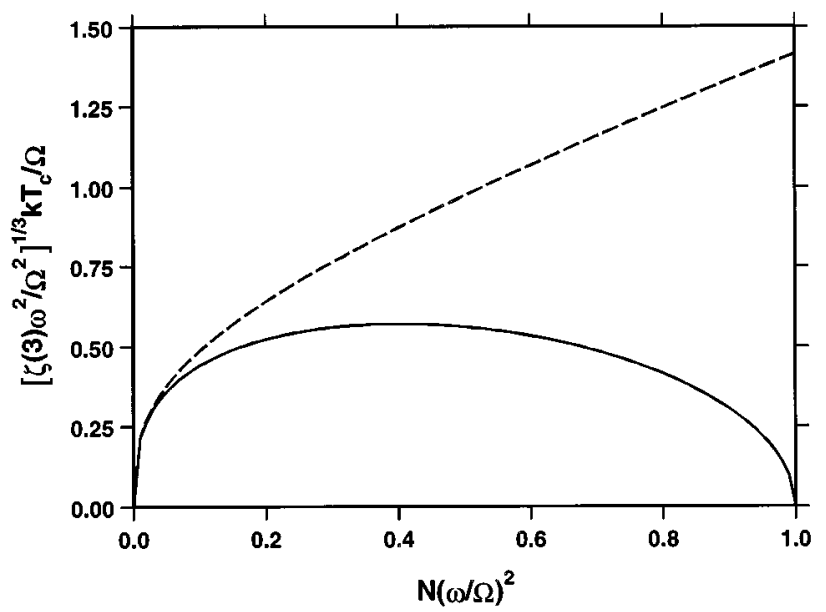

FIG. 4. Critical temperature $T_{c}$ as a function of the number of particles $N$ for a repulsive (full line) and an attractive (dashed line) two-body interaction.

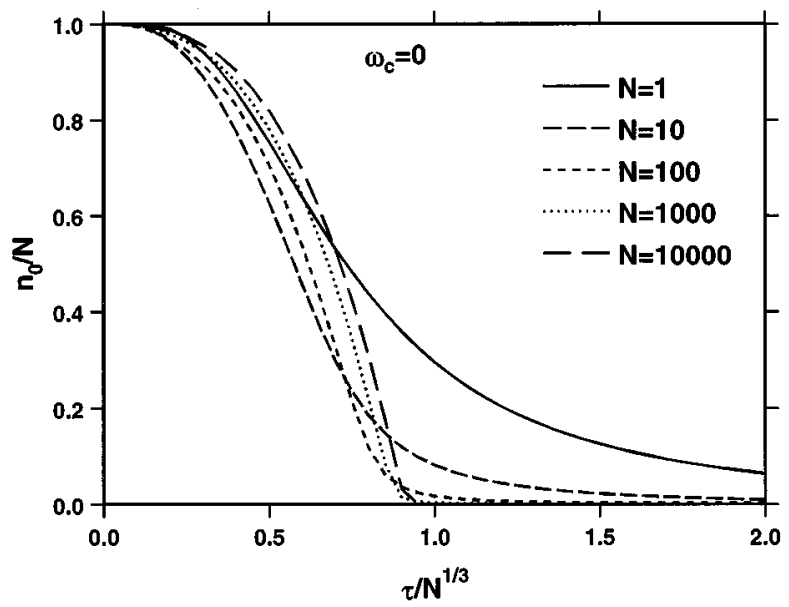

FIG. 5. Relative ground-state occupancy $n_{0} / N$ as a function of $\tau / N^{1 / 3}$, with $\tau$ the reduced temperature $\tau=k T / w$ for $\omega_{c}=0$ and for several values of $N$.

anisotropy only moderately influences the temperature dependence of the ground-state occupancy.

The internal energy $U_{\omega_{c}}=\partial\left(\beta G_{\omega_{c}}\right) / \partial \beta-\mu N$ becomes

$$
\begin{aligned}
U_{\omega_{c}}= & \sum_{l=1}^{\infty} \frac{\alpha^{l}}{D_{l}}\left(\Omega \frac{e^{-l \beta \Omega}}{1-e^{-l \beta \Omega}}+\left(s+\frac{1}{2} \omega_{c}\right) \frac{e^{-l \beta\left[s+(1 / 2) \omega_{c}\right]}}{1-e^{-l \beta\left[s+(1 / 2) \omega_{c}\right]}}\right. \\
& \left.+\left(s-\frac{1}{2} \omega_{c}\right) \frac{e^{-l \beta\left[s-(1 / 2) \omega_{c}\right]}}{1-e^{-l \beta\left[s-(1 / 2) \omega_{c}\right]}}\right)
\end{aligned}
$$

and its numerical evaluation once $\alpha$ is determined presents no numerical difficulties. The resulting specific heat is shown in Fig. 7 for $\omega_{c} / \Omega=5$. A comparison with Fig. 3 reveals that the anisotropy due to the magnetic field essentially broadens the peak in the specific heat near the condensation temperature, but does not substantially alter the structure.

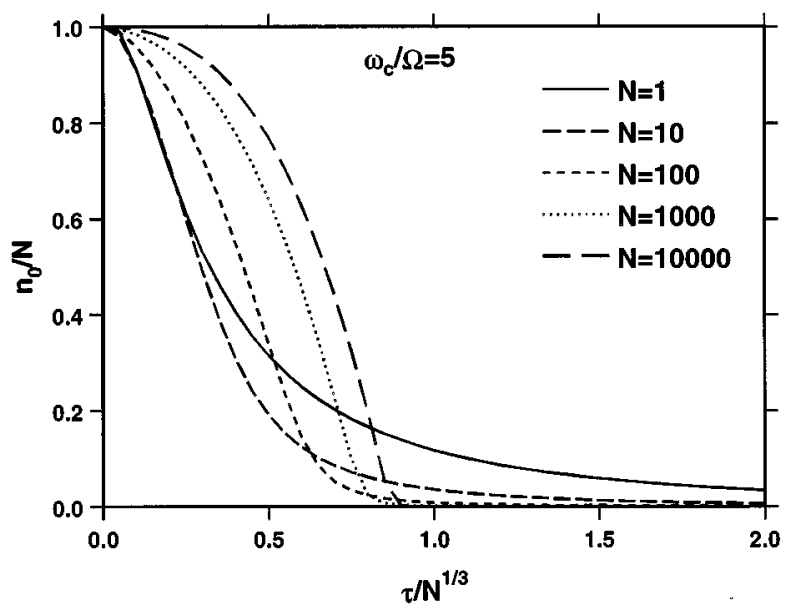

FIG. 6. Relative ground-state occupancy $n_{0} / N$ as a function of $\tau / N^{1 / 3}$ with $\tau$ the reduced temperature $\tau=k T / w$ for $\omega_{c} / \Omega=5$ and for several values of $N$. 


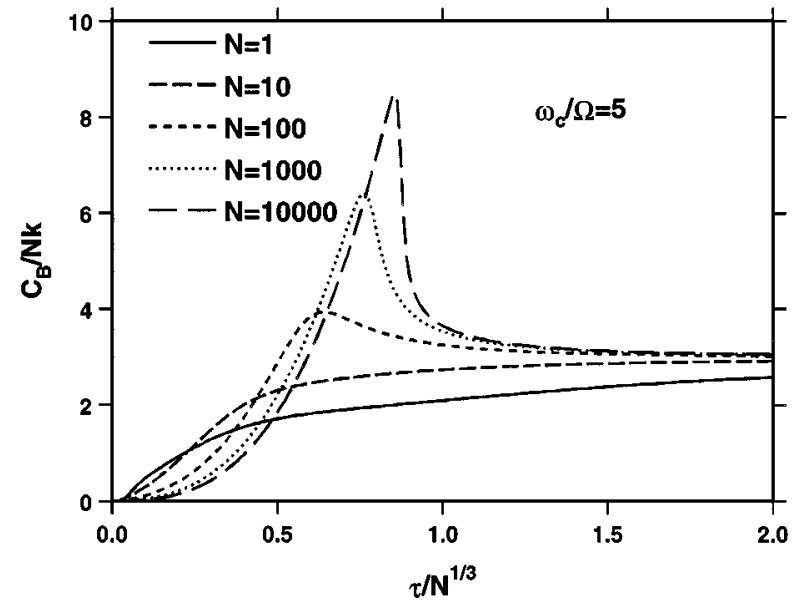

FIG. 7. Specific heat $C_{B} / N k$ per particle in units of the Boltzmann constant $k$ as a function of $\tau / N^{1 / 3}$ with $\tau$ the reduced temperature $\tau=k T / w$ for $\omega_{c} / \Omega=5$ and for several values of $N$.

\section{DISCUSSION AND CONCLUSION}

In this paper we applied the method of symmetrical density matrices, developed by Feynman for a system of noninteracting particles in a box, to a system of harmonically interacting particles in a confining parabolic potential. The interaction could be taken into account, due to the Gaussian nature of the propagators, allowing integration over the configuration space. The symmetrization resulting from the projection of the propagators for distinguishable particles on the appropriate representation of the permutation group gives rise to a series that could be summed using the generating function technique. Without these generating functions the calculation has to be restricted to a limited number of particles $[6,7,23]$. Using them, not only could the grandcanonical partition function $\Xi(u)$ be obtained in the parameter range of the model where $\Xi(u)$ is well defined, but also the canonical partition functions $Z(N)$ for a given number $N$ of identical particles could be obtained as a recursion of partition functions of a smaller number of particles for the interacting system. The recurrence relation for the activity $\rho_{N}$, i.e., the proportionality factor between $Z(N)$ and $Z(N-1)$, allows for an accurate numerical treatment of the thermodynamical quantities of the model, such as the internal energy and the specific heat.

Also the thermodynamic properties of the same model in the presence of a homogeneous magnetic field could be investigated along these lines. A detailed analysis of the ideal gas in a confining parabolic potential with anisotropy due to a magnetic field was presented; special attention was paid to the relation between the number of condensed atoms, the magnetic field, the strength of the confinement potential, and the total number of particles. The relationship between the parameters of our model and the characteristics of atomic traps [2-4] lies beyond the scope of the present paper.

It should be mentioned that in the absence of two-body interactions, the generating function can be identified as the grand-canonical partition function. In that case the specific heat as obtained from the grand-canonical ensemble for an average number of $\langle N\rangle$ particles calculated using the chemical potential equals the specific heat obtained from the canonical ensemble with the number of particles $N$ given by $\langle N\rangle$. For a more elaborate discussion we refer to [17].

It should also be mentioned that in this the model it is assumed that the spin degrees of freedom are fixed. This simplifying assumption is imposed by the symmetrization method, which becomes more involved if the spin degrees of freedom depend on the configuration of the particles.

In summary, the partition function of a general Gaussian model for bosons and fermions, with or without a magnetic field, has been calculated analytically and the thermodynamical properties of this model have been studied. Particular attention has been given to the Bose-Einstein condensation in the presence of two-body interactions, repulsive and attractive, and in the presence of a magnetic field.

\section{ACKNOWLEDGMENTS}

Part of this work was performed in the framework of the NFWO Projects Nos. 2.0093.91, 2.0110.91, G.0287.95, and WO.073.94N (Wetenschappelijke Onderzoeksgemeenschap, Scientific Research Community of the NFWO on "LowDimensional Systems") and in the framework of the European Community Program Human Capital and Mobility through Contracts Nos. CHRX-CT93-0337 and CHRXCT93-0124. F.B. acknowledges the National Fund for Scientific Research for financial support.
[1] R. P. Feynman, Statistical Mechanics, A Set of Lectures (Benjamin, Reading, MA, 1972).

[2] M. H. Anderson, J. R. Ensher, M. R. Matthews, C. E. Wieman, and E. A. Cornell, Science 269, 198 (1995).

[3] C. C. Bradlet, C. A. Sacket, J. J. Tollett, and R. G. Hulet, Phys. Rev. Lett. 75, 1687 (1995).

[4] K. B. Davis, M. O. Mewes, M. R. Andrews, N. J. van Druten, D. S. Durfee, D. M. Kurn, and W. Ketterle, Phys. Rev. Lett. 75, 3969 (1995).

[5] N. F. Johnson and M. C. Payne, Phys. Rev. Lett. 67, 1157 (1991).
[6] R. W. Haase and N. F. Johnson, Phys. Rev. B 48, 1583 (1993).

[7] W. Häusler, Z. Phys. B 99, 551 (1996).

[8] L. F. Lemmens, F. Brosens, and J. T. Devreese, Phys. Lett. A 189, 437 (1994).

[9] F. Brosens, J. T. Devreese, and L. F. Lemmens, Solid State Commun. 96, 137 (1995).

[10] L. F. Lemmens, F. Brosens, and J. T. Devreese, Phys. Rev. E 53, 4467 (1996).

[11] R. P. Feynman and A. R. Hibbs, Quantum Mechanics and Path Integrals (McGraw-Hill, New York, 1965).

[12] R. P. Feynman and H. Kleinert, Phys. Rev. A 34, 5080 (1986). 
[13] R. Giachetti and V. Tognetti, Phys. Rev. Lett. 55, 912 (1985).

[14] G. W. Ford, M. Kac, and P. Mazur, J. Math. Phys. 6, 504 (1961); see also Ref. [21].

[15] G. Röpstorff, Path Integral Approach to Quantum Mechanics (Springer, Berlin, 1994).

[16] R. P. Feynman, Phys. Rev. 84, 108 (1951).

[17] F. Brosens, J. T. Devreese, and L. F. Lemmens, Solid State Commun. 100, 123 (1996).

[18] S. Grossmann and M. Holthaus, Phys. Lett. A 208, 188 (1995).
[19] K. Kirsten and D. J. Toms, Report No. http://xxx.lanl.gov/ archive/cond-mat/9607047.

[20] M. Takahashi and M. Imada, J. Phys. Soc. Jpn. 53, 963 (1984).

[21] E. Lieb and D. Mattis, Mathematical Physics in One Dimension (Academic, New York, 1966).

[22] B. Simon, Functional Integration and Quantum Physics (Academic, New York, 1979).

[23] M. Hammermesh, Group Theory and its Application to Physical Problems (Dover, New York, 1989). 